\title{
SLC34A3 Intronic Deletion in a New Kindred with Hereditary Hypophosphatemic Rickets with Hypercalciuria
}

\author{
Shirin Hasani-Ranjbar, Mahsa M. Amoli, Azadeh Ebrahim-Habibi, Ehsan Dehghan, Akbar Soltani, \\ Parvin Amiri, Bagher Larijani \\ Tehran University of Medical Sciences, Endocrinology and Metabolism Research Institute, Tehran, Iran
}

\begin{abstract}
Objective: Hereditary hypophosphatemic rickets with hypercalciuria (HHRH) is an autosomal recessive form of hypophosphatemia with hyperphosphaturia, hypercalciuria, and hypercalcemia. In two reports on six affected kindreds with HHRH, the disease was mapped to chromosome 9q34, which contains the SLC34A3 gene that encodes the renal type $2 C$ sodium-phosphate cotransporter. Our objective was to define the clinical course of these cases in a family with HHRH and to screen for SLC34A3 gene in order to determine whether these mutations are responsible for HHRH.

Methods: After clinical and biochemical evaluations, the entire SLC34A3 gene was screened using PCR amplification followed by direct sequencing technique. In this paper, we describe a new kindred with HHRH and a case of progressive and complicated HHRH presenting at age 27 years.

Results: We found 101-bp deletion in intron 9 of the SLC34A3 gene. The index patient was homozygous for this mutation which has been previously reported in a Caucasian population. This is the first report for presence of SLC34A3 intron 9 deletion in an Iranian population.

Conclusions: These data showed that HHRH can be easily missed or underdiagnosed. Genetic evaluation of patients with familial hypercalciuria, hypophosphatemia and nephrolithiasis is needed for further information on the prevalence and management of this rare disorder.

Key words: Hypophosphatemia, hypercalciuria, hereditary hypophosphatemic rickets with hypercalciuria, nephrocalcinosis, SLC34A3 gene
\end{abstract}

Conflict of interest: None declared

Received: 13.01.2012

Accepted: 14.03 .2012

\section{Introduction}

Phosphorus is a mineral essential for numerous cellular functions and is an important constituent of bones. Hypophosphatemia in children leads to rickets resulting in poor growth and frequently in skeletal deformities. Among the various causes of low serum phosphorus are inherited disorders associated with increased urinary excretion of phosphate, which include X-linked hypophosphatemia $(X L H)$, autosomal recessive hypophosphatemic rickets (ARHR), autosomal dominant hypophosphatemic rickets (ADHR), and hereditary hypophosphatemic rickets with hypercalciuria $(\mathrm{HHRH})$ (1). Clinical and biological characteristics of hypophosphatemia, as well as its management, depend on the specific etiology (2). $\mathrm{HHRH}$ is an autosomal recessive form of hypophosphatemia that has been recently described with hyperphosphaturia, hypercalciuria, hypercalcemia, decreased serum parathormone (PTH) levels and increased serum alkaline phosphatase (ALP) activity $(3,4,5,6)$. In addition to cases from a Bedouin tribe and a kindred of Jewish Yemenite origin, a few sporadic cases of this disease have also been reported $(5,6,7,8,9,10)$. In two reports on six kindreds affected by $\mathrm{HHRH}$, the disease was mapped to chromosome 9q34, which contains the SLC34A3 gene that encodes the renal type 2c sodium-phosphate cotransporter (10). We describe here a new kindred with $\mathrm{HHRH}$. The index subject was a man with progressive and complicated $\mathrm{HHRH}$, who presented to our clinic at age 27 years, with no prior treatment.

Address for Correspondence

Shirin Hasani-Ranjbar MD, Endocrinology and Metabolism Research Institute, $5^{\text {th }}$ Floor, Shariati Hospital, North Kargar Ave., Tehran 14114, Iran Phone: +982188220037 Fax: +982188220052 E-mail: shirinhasanir@yahoo.com

(๑) Journal of Clinical Research in Pediatric Endocrinology, Published by Galenos Publishing. 


\section{Methods}

\section{Patients and data collection}

The family (Figure 1) was evaluated for past medical history. The patient's physical examination and biochemical assessment were performed in the endocrine unit of the Shariati Hospital, Tehran University of Medical Sciences. Blood samples were taken after a 12-hour overnight fast. In addition, urine was collected for $24 \mathrm{~h}$ to measure calcium, creatinine and phosphorus. Tubular reabsorption of phosphate (TRP) was calculated using the following formula: 1 - (urine phosphorus $X$ serum creatinine/serum phosphorus $X$ urine creatinine). Then TMP/GFR (tubular maximum reabsorption threshold of phosphate per glomerular filtration rate) was calculated using a nomogram developed by Walton and Bijvoet $(1,10)$. Skeletal Xrays were taken, and renal ultrasonography was performed. Genetic analysis and protein modeling were also conducted.

\section{Genetic analysis}

Genomic DNA was isolated from peripheral blood leukocytes using the salting-out method. The entire SLC34A3 gene which spans approximately $5 \mathrm{~kb}$ of genomic DNA was amplified, followed by direct sequencing according to the assay described by Bergwitz et al (11).

\section{Protein modeling}

The sequence of sodium-phosphate cotransporter type 2c (gil25014088) was retrieved from NCBI protein (http://uww.ncbi.nlm.nih.gov/protein). Secondary structure

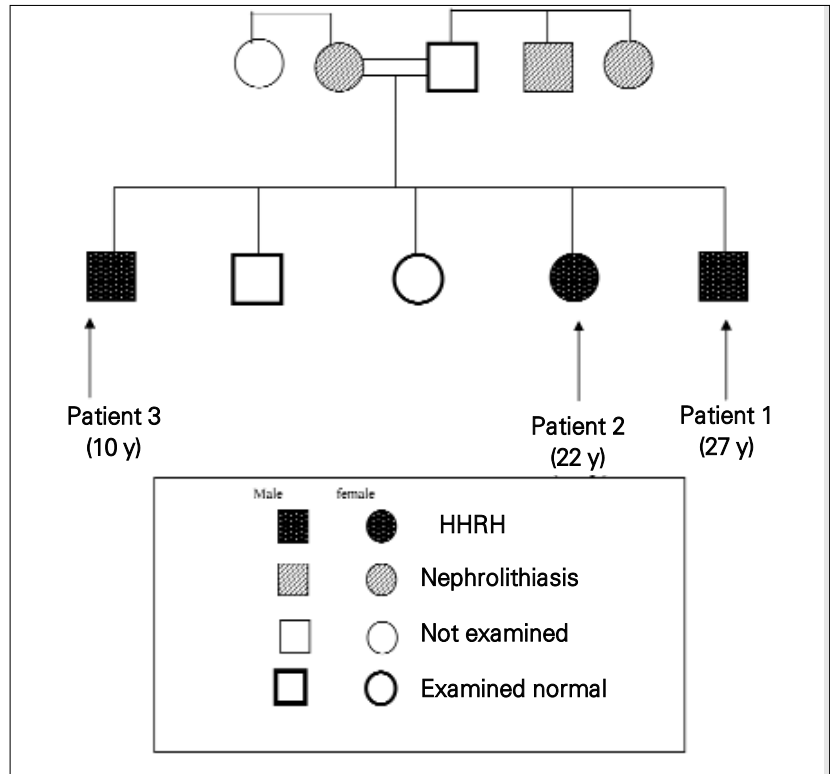

Figure 1. Genetic relationship of patients with hereditary hypophosphatemic rickets with hypercalciuria $(\mathrm{HHRH})$ and 3 subjects with hypercalciuria and renal stones prediction of the protein was performed with the use of the HMMTOP server (mww.enzim.hu/hmmtop) (12). The obtained predicted structure was schematically drawn with the help of TOPO2 (Johns SJ, TOPO2,Transmembrane protein display software, http://umw.sacs.ucsf.edu/TOPO2/). Translation of nucleotide to protein sequence was performed with the use of EXPASY translate tool (http://web.expasy.org/translate/).

\section{Results}

\section{Subjects}

Patient 1 (Index patient, Figure 1). A 27-year-old man, whose parents were distant relatives, was referred to our clinic for evaluation of skeletal deformities, bone pain and nephrocalcinosis. He presented with leg deformities and a history of progressive departure from normal growth starting at age 10 years. At age 12 years, he had undergone an operation because of bladder and kidney stones, and a second surgical intervention had been performed at age 25 years. The patient also had a history of early dental caries. By age 18 years, his physical activity had become very restricted. He had no drug addictions. He had received courses of antibiotic therapy for recurrent urinary tract infections.

At presentation, muscle weakness was not a prominent feature. The patient's height was $110 \mathrm{~cm}$ (SD score: -9.0) and his weight was $50 \mathrm{~kg}$. He displayed striking deformities in his chest and in his upper and lower limbs. Laboratory data revealed hypophosphatemia, elevated ALP activity and hypercalciuria. TMP/GFR was $1.4 \mathrm{mg} / \mathrm{dL}$ and 24-hour phosphate excretion was $1080 \mathrm{mg}$ (Table 1). Urine culture was positive for Escherichia coli.

Radiography of the limbs revealed late complications of rickets (Figure 2). Renal ultrasonography showed that the right kidney size was $77 \times 33 \mathrm{~mm}$ and the left kidney size was

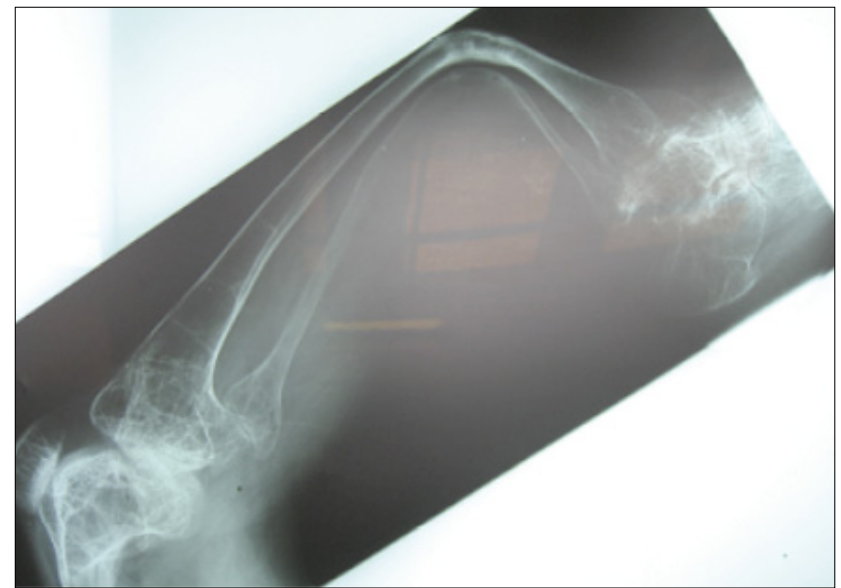

Figure 2. Radiograph of the right leg showing profound loss of bone with severe bending of the bone shafts and thin cortical layer 
$111 \times 50 \mathrm{~mm}$. There were two renal stones in each kidney. No signs of hydronephrosis were noted.

After evaluation, the patient was treated with neutral phosphate only. Two gram of elemental phosphorus per day

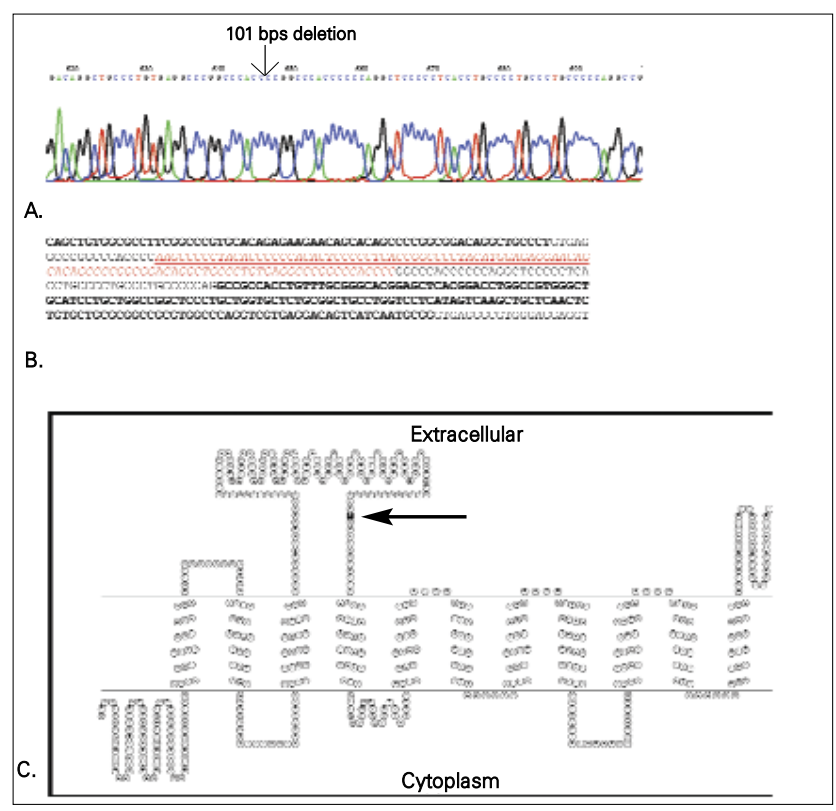

Figure 3. Mutation analysis of SLC34A3 gene and protein model. The segment of the chromatogram with arrow indicates the site of deletion in SCL34A3 gene in our patient was given orally in 3 divided doses. Bone pains disappeared within three weeks. Serum calcium decreased and reached a near-normal level. Serum phosphorus level (in fasting state, 2 hours after the first dose of neutral phosphate) increased toward normal. TMP/GFR remained unchanged.

Patient 2 was a 22-year-old male (Figure 1) who had short stature (SD score:-4.5), skeletal deformities and nephrolithiasis. The results of laboratory tests are shown in Table 1.

Patient 3 (Figure 1) was a 10-year-old boy who also had bone deformities and renal calculi. He was diagnosed in another clinic and his laboratory data were not available. His family history was positive for nephrolithiasis in his mother, maternal uncle and aunt and they were all under treatment with thiazide.

\section{Genetic analysis}

Mutation analysis revealed presence of 101-bp deletion in intron 9. The index patient was homozygous for this mutation. Figure 3 illustrates the presence of deletion in our patient.

\section{Protein modeling}

Deletion of the indicated segment in intron 9 (Figure 3B) has been experimentally shown to result in intron retention in the mRNA (13). The 22 amino acids that would result from this insertion have been found to have the following sequence: EARPTPAHPPGSPSPAPALPPG. Since no three-dimensional structure has been reported for this protein or closely related ones, a secondary structure prediction was performed in order

Table 1. Laboratory- test results of two patients with HHRH

\begin{tabular}{lccc}
\hline Variable & Reference range (Adult) & Patient 1 (weight 50kg) & Patient 2 (weight 49kg) \\
\hline Urea nitrogen (mg/dl) & $(7.0-18.0)$ & 10 & 7.1 \\
Serum creatinine (mg/dl) & $(0.3-1.40)$ & 1 & 0.8 \\
Serum calcium (mg/dl) & $(8.5-10.5)$ & 10.5 & 9.5 \\
Serum phosphorus (mg/dl) & $(2.5-4.5)$ & 2.4 & 2.9 \\
Alkaline phosphatase (u/liter) & (Female:64-306) & 855 & 466 \\
Parathyroid hormone (pg/ml) & (Male:80-306) & 25 & 14 \\
Serum 25-hydroxy vitamin D (ng/ml) & $10-62$ & 50 & - \\
Urine creatinine (mg 24h) & $20-100$ & 1000 & 440 \\
Urine calcium (mg/24h) & $600-1800$ & 540 & 358 \\
& Up to 300 & & \\
Urine phosphorus (mg/24h) & (Up to 4 mg/kg) & 1080 & 676 \\
TRP & - & 0.55 & 1.4 \\
TMP/GFR(mg/dl) & $0.85-0.98$ & 1.4 & 1.7 \\
\hline
\end{tabular}

To convert the values for calcium to millimoles per liter, multiply by 0.250 to convert the values for phosphorus to millimoles per liter, multiply by 0.3229 to convert the values for 25- $\mathrm{OH}$ vitamin $\mathrm{D}$ to nanomoles per liter, multiply by 2.496 .

Tubular reabsorbtion of phosphate (TRP). TRP is calculated using the following formula: 1- (urine phosphorus $\mathrm{x}$ seum creatinine / serum phosphorus $\mathrm{x}$ urine creatinine). Tubular threshold maximum for phosphorus per glumerular filtration rate (TMP/GFR) can be calculated using a nomogram developed by Walton and Bijvoet $(1,10)$. HHRH: hereditary hypophosphatemic rickets with hypercalciuria 
Hasani-Ranjbar S et al.

SLC34A3 Intronic Deletion in HHRH

to get an insight into the putative position of the added residues. The results obtained from the HMMTOP server were used, based on the fact that this algorithm was observed to predict a more correct topology for the similar sodiumphosphate cotransporter lla (from rat) (14), compared with other existing algorithms. The hypothetical representation of sodiumphosphate cotransporter $2 c$ topology has been drawn with the use of TOPO2, and contains 11 transmembrane segments (Figure $3 \mathrm{C}$ ). The putative addition of amino acids would occur after C309, whose position is indicated in Figure 3C.

\section{Discussion}

It should be emphasized that the index patient (patient 1) manifested severe short stature and bowed legs, and the biochemical assessment revealed hypophosphatemia, phosphaturia and calciuria. As a matter of fact, hereditary forms of hypophosphatemic rickets that are associated with renal phosphate loss include $\mathrm{X}$-linked and autosomal dominant disease as well as $\mathrm{HHRH}$. The latter disease, the only form that can explain hypercalciuria, is very rare $(1,2)$. The etiologies of early-onset nephrocalcinosis in consanguineous families include five major inherited recessive disorders, including $\mathrm{HHRH}$ (3). The development of nephrocalcinosis in X-linked hypophosphatemic rickets is probably related to intermittent episodes of hypercalcemia and hypercalciuria. These can result from an excessive calcitriol dose or from noncompliance with oral phosphate supplementation and is associated with the dose of phosphate received $(4,15)$. On the other hand, in patients with $\mathrm{HHRH}$ due to hypophosphatemia, an appropriate elevation in the serum levels of 1,25-dihydroxyvitamin $D$ leading to hypercalciuria, hypercalcemia and decreased serum PTH levels and increased serum ALP activity are noted $(5,6)$. Elevated ALP is due to hypophosphatemia itself. These biochemical features were observed in two of our patients. Most HHRH patients presenting with rickets have short stature and increased renal phosphate clearance (low TMP/GFR) (1). In homozygous state (as in our index patient) or in compound heterozygoty, urinary loss of phosphorus is more significant, resulting in low serum phosphorus and rickets (16). However, milder forms may be underdiagnosed if the hypophosphatemia is mild; such patients present with hypercalciuria and nephrolithiasis, but no signs of bone disease are observed (5). These heterozygous patients have renal stones without rickets (16).

$\mathrm{HHRH}$ was initially described as a new syndrome in a large Bedouin tribe kindred $(7,8)$. Up to now, only a few cases including a kindred of Jewish Yemenite origin as well as familial and sporadic cases from Europe, North America and Japan have been reported $(7,8,9,10)$. We described here $\mathrm{HHRH}$ in an Iranian family. These family members (some with progressive $\mathrm{HHRH}$ ) were diagnosed in another health facility as familial nephrolithiasis. They were also advised not to use calcium, phosphate and vitamin D supplementation. These findings lead us to think that $\mathrm{HHRH}$ is a condition that is underdiagnosed possibly due to its similarity to other hypophosphatemic syndromes or familial nephrolithiasis in its clinical, radiological and most biochemical parameters. Patients with the hypercalciuric form of hypophosphatemic rickets should be treated with phosphorus supplementation alone (1). The addition of active vitamin $D$ metabolites could cause deterioration in the patient's condition (7). In skeletally immature patients, the goals of therapy are attainment of a normal longitudinal growth rate and achievement of normal serum ALP concentrations, rather than normalization of serum phosphate concentration. On the other hand, in skeletally mature patients, the goal of therapy is to prevent the symptoms of hypophosphatemia $(7,17)$. We should also stress, as also noted in our patient and his family who had to undergo operation because of kidney stones, that kidney stones and refractory urinary tract infections are important complications of the disease.

$\mathrm{HHRH}$ is inherited in an autosomal recessive manner (18). The disorder is known to be caused by genetic mutations of the renal type $2 \mathrm{c}$ sodium-phosphate cotransporter. In two reports on six affected kindreds with $\mathrm{HHRH}$, the disease was mapped to chromosome 9q34, which contains the SLC34A3 gene that encodes the renal type $2 \mathrm{c}$ sodium-phosphate cotransporter $(10,13,19,20,21)$. However, the correlation between gene abnormalities and osteoblastic function in human $\mathrm{HHRH}$ osteoblasts is still unclear (9). Genetic analysis in our study showed a previously reported deletion in intron 9 in the $S L C 34 A 3$ gene. This is the first report for presence of this mutation in an Iranian patient. It has been suggested that the presence of identical sequence repeats in this region might have endorsed misalignment during meiosis causing such a deletion. Large region deletions in small introns are not frequently reported in other conditions. As the resulting mRNA was observed to contain the truncated intron with $66 \mathrm{bp}$ (13), a putative addition of 22 amino acids may occur in the potentially produced protein. In this case, the segment would be located in the second extracellular loop of the protein (Figure $3 \mathrm{C}$ ), probably affecting the correct folding of the protein afterwards, resulting in a nonfunctional product. Even though this extra cellular loop is of a large size and should probably be able to accommodate for smaller insertions, in this case, addition of a 22-amino acid segment would result in too much change in the consecutive membrane segments. $\mathrm{HHRH}$ is an autosomal recessive disease and our patient in this study was 
homozygous for SLC34A3 gene mutation, therefore, this patient must have inherited the mutation from both parents for complete disease manifestations. However, the presence of nephrolithiasis in the family members might indicate some related clinical phenotype occurring in the presence of one mutated allele.

Our data has also shown that $\mathrm{HHRH}$ can be easily missed or underdiagnosed. Genetic evaluation of patients with familial hypercalciuria, hypophosphatemia and nephrolithiasis are needed for further information on the prevalence and management of this rare disorder.

\section{Acknowledgments}

We gratefully thank the patients and their family for consenting to the publication of this study.

\section{References}

1. Bastepe $M$, Jüppner $H$. Inherited hypophosphatemic disorders in children and the evolving mechanisms of phosphate regulation. Rev Endocr Metab Disord 2008;9:171180.

2. Vélayoudom-Céphise $\mathrm{FL}$, Vantyghem $\mathrm{MC}$, Wémeau JL. Hereditary hypophosphatemia in adults. Presse Med 2005:34:1720-1726.

3. Vargas-Poussou R, Cochat P, Le Pottier N, Roncelin I, Liutkus A, Blanchard A, Jeunemaître X. 2008 Report of a family with two different hereditary diseases leading to early nephrocalcinosis. Pediatr Nephrol 2008;23:149-153.

4. Tieder M, Blonder J, Strauss S, Shaked U, Maor J, Gabizon D, Manor $H$, Sela BA. Hyperoxaluria is not a cause of nephrocalcinosis in phosphate-treated patients with hereditary hypophosphatemic rickets. Nephron 1993;64:526-531.

5. Tieder M, Modai D, Samuel R, Arie R, Halabe A, Bab I, Gabizon D, Liberman UA. Hereditary hypophosphatemic rickets with hypercalciuria. N Engl J Med 1985;312:611-617.

6. Gazit D, Tieder $M$, Liberman UA, Passi-Even L, Bab IA Osteomalacia in hereditary hypophosphatemic rickets with hypercalciuria: a correlative clinical-histomorphometric study. $\mathrm{J}$ Clin Endocrinol Metab 1991;72:229-235.

7. Tieder M, Arie R, Bab I, Maor J, Liberman UA. A new kindred with hereditary hypophosphatemic rickets with hypercalciuria: implications for correct diagnosis and treatment. Nephron 1992;62:176-181.

8. Tieder M, Modai D, Shaked U, Samuel R, Arie R, Halabe A Maor J, Weissgarten J, Averbukh Z, Cohen N, Edelstein S, Liberman UA. "Idiopathic" hypercalciuria and hereditary hypophosphatemic rickets. Two phenotypical expressions of a common genetic defect. N Engl J Med 1987;316:125-129.

9. Yamamoto T, Michigami T, Aranami F, Segawa H, Yoh K, Nakajima S, Miyamoto K, Ozono K. Hereditary hypophosphatemic rickets with hypercalciuria: a study for the phosphate transporter gene type IIc and osteoblastic function. J Bone Miner Metab 2007;25:407-413.
10. Walton RJ, Bijvoet OL. Nomogram for derivation of renal threshold phosphate concentration. Lancet 1975;2:309-310.

11. Bergwitz C, Roslin NM, Tieder M, Loredo-Osti JC, Bastepe M, Abu-Zahra H, Frappier D, Burkett K, Carpenter TO, Anderson D, Garabedian M, Sermet I, Fujiwara TM, Morgan K, Tenenhouse HS, Juppner H. SLC34A3 mutations in patients with hereditary hypophosphatemic rickets with hypercalciuria predict a key role for the sodium-phosphate cotransporter $\mathrm{NaPi}$-llc in maintaining phosphate homeostasis. Am J Hum Genet 2006;78:179-192.

12. Tusnady GE, Simon I. The HMMTOP transmembrane topology prediction server. Bioinformatics 2001;17:849-850.

13. Ichikawa S, Sorenson AH, Imel EA, Friedman NE, Gertner JM, Econs MJ. Intronic deletions in the SLC34A3 gene cause hereditary hypophosphatemic rickets with hypercalciuria. J Clin Endocrinol Metab 2006;91:4022-4027.

14. Radanovic T, Gisler SM, Biber J, Murer H. Topology of the Type $l \mathrm{la} \mathrm{Na}+\mathrm{Pi}$ Cotransporter. J Membrane Biol 2006;212:41-49.

15. Verge CF, Lam A, Simpson JM, Cowell CT, Howard NJ, Silink $M$. Effects of therapy in X-linked hypophosphatemic rickets. N Engl J Med 1991;325:1843-1848.

16. Tiosano D, Hochberg Z. Hypophosphatemia: the common denominator of all rickets. J Bone Miner Metab 2009;27: 392401.

17. Lau YK, Wasserstein A, Westby GR, Bosanac P, Grabie M, Mitnick P, Slatopolsky E, Goldfarb S, Agus ZS. Proximal tubular defects in idiopathic hypercalciuria: resistance to phosphate administration. Miner Electrolyte Metab 1982;7:237-249.

18. Gambaro G, Vezzoli G, Casari G, Rampoldi L, D'Angelo A, Borghi L. Genetics of hypercalciuria and calcium nephrolithiasis: from the rare monogenic to the common polygenic forms. Am J Kidney Dis 2004;44:963-986.

19. Lorenz-Depiereux B, Benet-Pages A, Eckstein G, Tenenbaum-Rakover $Y$, Wagenstaller J, Tiosano D, GershoniBaruch R, Albers N, Lichtner P, Schnabel D, Hochberg Z, Strom TM. Hereditary hypophosphatemic rickets with hypercalciuria is caused by mutations in the sodiumphosphate cotransporter gene SLC34A3. Am J Hum Genet 2006;78:193-201.

20. van den Heuvel L, Op de Koul K, Knots E, Knoers N, Monnens L. Autosomal recessive hypophosphataemic rickets with hypercalciuria is not caused by mutations in the type II renal sodium/phosphate cotransporter gene. Nephrol Dial Transplant 2001;16:48-51.

21. Jones A, Tzenova J, Frappier D, Crumley M, Roslin N, Kos C, Tieder $\mathrm{M}$, Langman $\mathrm{C}$, Proesmans $\mathrm{W}$, Carpenter $\mathrm{T}$, Rice $\mathrm{A}$, Anderson D, Morgan K, Fujiwara T, Tenenhouse H. Hereditary hypophosphatemic rickets with hypercalciuria is not caused by mutations in the Na/Pi cotransporter NPT2 gene. J Am Soc Nephrol 2001;12:507-514. 